\title{
Validating genomic tools for precision medicine in CLL: ERIC leads the way
}

\author{
Adrian Wiestner \\ National Heart, Lung, and Blood Institute, National Institutes of Health, Bethesda, MD, USA \\ E-mail: ADRIAN WIESTNER - wiestnera@mail.nih.gov
}

doi:10.3324/haematol.2020.270652

C2021 NIH (National Institutes of Health)

I $\mathrm{n}$ this issue of Haematologica, Sutton and colleagues report the comparative analysis of targeted next-generation sequencing (NGS) panels for the detection of gene mutations in chronic lymphocytic leukemia (CLL). ${ }^{1}$ This important contribution to the standardization of mutation profiling for clinical practice was made possible by a multicenter study conducted by the European Research Initiative on CLL (ERIC).

CLL is characterized by the clonal expansion of mature $B$ cells in blood, bone marrow, and lymph nodes. ${ }^{2,3}$ CLL has a highly variable clinical course. While some patients may never develop symptomatic disease and do not require treatment, others can progress rapidly and may need repeat lines of therapy. Relapsing disease often carries additional genetic abnormalities that contribute to progression and/or treatment resistance. Determination of the mutational status of the rearranged immunoglobulin heavy chain variable (IGHV) genes contributes greatly to the dissection of disease heterogeneity. IGHV sequences with $\geq 98 \%$ homology to germline are considered unmutated. Compared to CLL with mutated IGHV genes, IGHV unmutated CLL is associated with more rapidly progressive disease, early relapse, and more frequent acquisition of additional genetic lesions. IGHV mutation status is therefore one of the most important prognostic factors in CLL. Fluorescence in situ hybridization (FISH) cytogenetics detect additional well-validated prognostic markers. Deletion of chromosomal arms 17p (del17p) or 11q (del11q) are associated with shorter overall survival and early progression after treatment with chemoimmunotherapy. Dependencies of the clonal cells on B-cell receptor signaling and BCL2 are now specifically targeted by kinase inhibitors and venetoclax, respectively. Having demonstrated superior disease control compared to chemoimmunotherapy in randomized trials, these targeted agents have become preferred treatment options for patients with CLL. ${ }^{3}$

Over the last years, genome-wide analyses using NGS contributed a large number of putative driver mutations as well as mutations associated with treatment resistance. Aggregating data from two large NGS studies covering approximately 1,000 patient samples, Lazarian et al. counted 75 significantly mutated genes. ${ }^{4}$ Thirty-five were found at a frequency $>1 \%$ in the combined data set, and 28 were common to both studies. However, most gene mutations were present in $<5 \%$ of patients. The most commonly mutated genes function in DNA damage response and cell cycle control (ATM, TP53, POT1), RNA and ribosomal processing (SF3B1, XPO1, RPS15), NOTCH1 signaling (NOTCH1), and immunoreceptor signaling (BIRC3, MYD88, EGR2). ${ }^{2,4}$ Several of the somatic mutations have been validated as independent prognostic markers. In most patients, the tumor cells carry more than one putative driver mutation often in combination with one or more chromosomal aberration detected by FISH. Integrating somatic mutations and FISH cytogenetics improves the separation of risk groups regarding overall survival. ${ }^{5}$
The increasing number of mutations and the complexity and heterogeneity of the tumor genome in CLL pose a need for techniques that can concurrently screen multiple genes. Targeted amplicon-based gene panels are widely pursued as an option that can meet the demands while being practical for routine testing. A number of technical options exist. The comparative performance of these different technologies in the hands of clinical laboratories is the focus of the study reported here. ${ }^{1}$ The coordinating ERIC site distributed tumor DNA from 48 CLL patients to six participating European centers. Three different amplicon-based targeted assays were used in the study: HaloPlex Target Enrichment System (Agilent Technologies), Illumina TruSeq Custom Amplicon (Illumina) and Multiplicom CLL Multiplex MASTR Plus (Agilent). Each assay was used by two centers allowing for a comparison of the performance of the individual assays. Paired-end sequencing was performed on Illumina MiSeq instruments. All sequencing data were centrally analyzed using a custom bioinformatics pipeline. Across all six centers, overall reproducibility of targeted NGS was assessed by looking at the inter-laboratory variation in detecting mutations. 107 of 115 mutations were detected in all six centers for a remarkable concordance of $93 \%$. A variant allele frequency (VAF) of $5 \%$ had to be reached in at least one center for the variant to be included in the concordance counts. Noteworthy, eight variants were detected in five of the six centers and missed in one. Six of theses eight variants concerned minor subclonal mutations present at low frequency. The full report is rich in detail on the performance of the individual assays and the reasons for false negatives and the rare false positives having VAF $\geq 5 \%$. Suffice it to say that all the assays performed well with high concordance between sister centers utilizing the same methodology. ${ }^{1}$

The concordance of $93 \%$ for inter-laboratory performance is based on mutations with VAF $\geq 5 \%$. VAF cutoffs of $10 \%$, the approximate detection limit of Sanger sequencing, are widely used in clinical routine. Despite advantages of NGS, reliable detection of low-level variants may be difficult. Pushing the limit of detection lower requires additional technical adaptions. In the ERIC study, the investigators explored high sensitivity assays containing unique molecular identifiers to detect low-level variants. ${ }^{1}$ While successful in principle, this method adds to complexity and will require additional efforts to implement in routine diagnostics.

Given the technical difficulties, do we really need to know about low frequency mutations? Yes, because, at least for some genes, low-level variants may be clinically relevant. In patients treated with chemoimmunotherapy, minor clones harboring TP53 mutations have been shown to expand by the time of relapse. ${ }^{6}$ The strong selective advantage conferred by TP53 mutations may explain observations that even minor clones harboring TP53 mutations (VAF <10\%) confer poor prognosis. ${ }^{7,8} \mathrm{Nadeu}$ and colleagues screened for known driver mutations in 28 genes. They identified some as having a prog- 


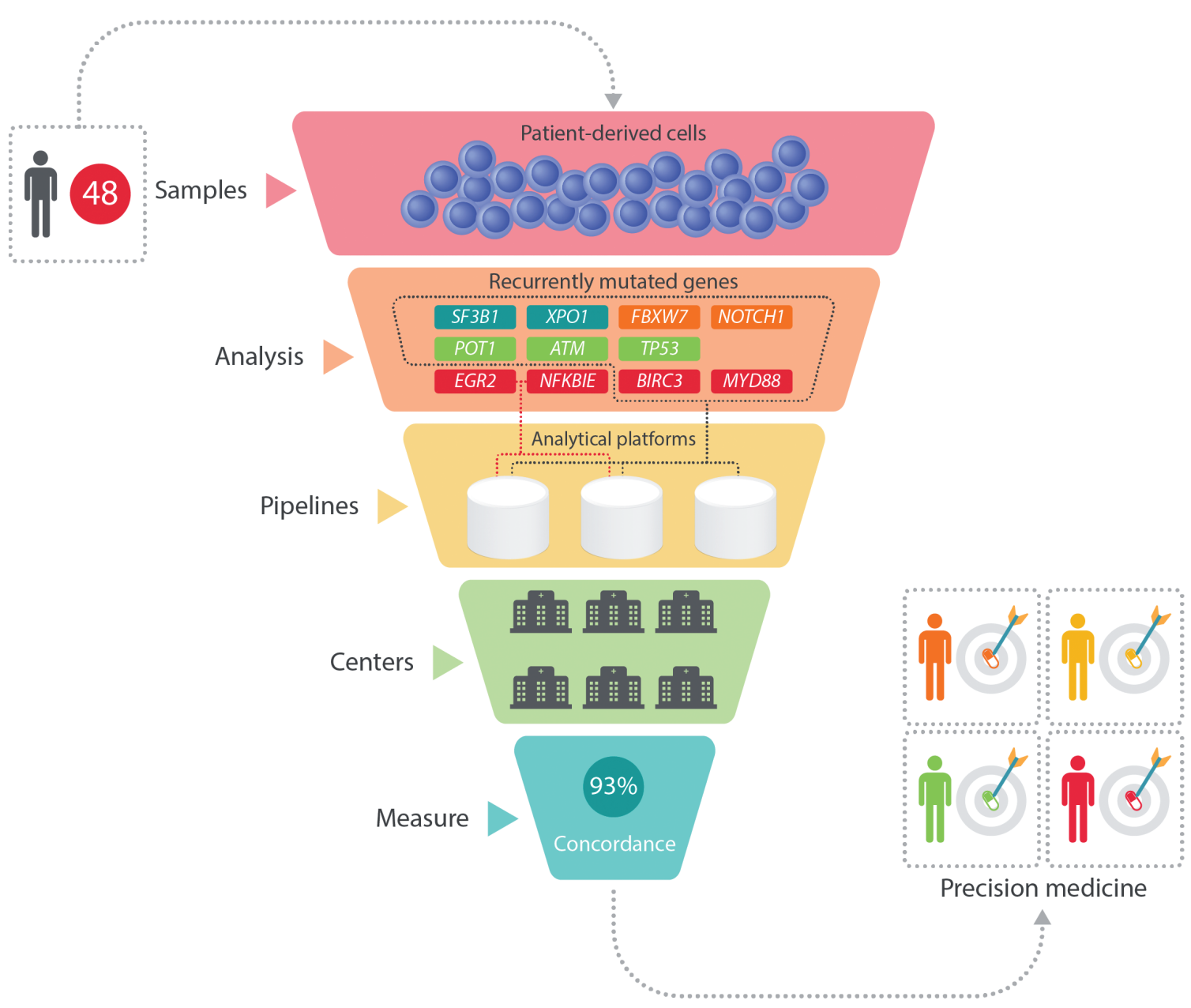

Figure 1. European multicenter evaluation of amplicon-based next generation sequencing assays.

nostic impact independent of VAF, for example NFKBIE, while for others, for example NOTCH1, only high but not low VAF was associated with inferior survival. ${ }^{9}$ Independent of specific prognostic information of a given variant, the mere presence of low VAF mutations reflects clonal complexity that, in turn, has been associated with shorter time to first treatment, ${ }^{9}$ and in patients treated with ibrutinib, with the risk of clonal evolution and eventual disease progression. ${ }^{10}$ Specific mutations in BTK or PLCG2 and BCL2 that are associated with disease progression on BTK inhibitors or venetoclax, respectively, may be detected at low VAF many months before overt clinical progression. ${ }^{11-13}$ While the presence of these mutations does not immediately warrant changes in treatment, they can identify patients that could benefit from closer observation.

This ERIC initiative takes an important step towards standardization of NGS based testing in clinic practice. A few considerations regarding widespread implementation may be in order. The common testing material consisted of DNA from 48 patients. ${ }^{1}$ Ideally, all mutations of interest, at variable VAF would be represented in the testing material which could present challenges to scale for widespread standardization. Bioinformatics was centralized. Using different bioinformatic pipelines could add addi- tional variability. All test sites are academic ERIC member institutions that are experienced with standardization efforts that ERIC has been conducting over many years. Conceivably, adding less experienced centers could lower the concordance rate. In localities where different commercial entities may be doing the bulk of testing the approach may also have to be adapted. Overall, the current study highlights once more the extraordinary contributions ERIC makes to the standardization of key laboratory data in CLL including IGHV sequencing, diagnostic flow cytometry, and the detection of minimal residual disease..$^{14-16}$

The performance of NGS assays reported here provides a solid basis for implementation in clinical testing. So the tools for precision medicine seem ready. Are we ready to use them? Given the expanding choices of therapies with distinct mechanisms of action, predictive markers that guide patients to the most promising therapy would be very valuable. However, to date, few predictive markers have been identified. IGHV unmutated status is predictive of benefit from targeted therapy with ibrutinib or venetoclax based therapy over chemoimmunotherapy. Mutated NOTCH1 was found to predict lack of benefit of adding rituximab to fludarabine and cyclophosphamide, but that provides little actionable information for current treat- 
ment choices. TP53 aberrations, del(17p) and/or mutated TP53, indicate benefit from treatment with novel agents over chemoimmunotherapy but also remain associated with earlier progression in patients treated with targeted therapy. ${ }^{17,18}$ In the CLL14 trial that compared venetoclax with obinutuzumab to chlorambucil with obinutuzumab, mutated BIRC3, and SF3B1 mutations were independent adverse factors for outcome in the chemoimmunotherapy arm but not in the venetoclax arm. ${ }^{17}$ While formally not identified as predictive markers, BIRC3 and SF3B1 mutations, as well as TP53 aberrations would still reasonably guide patients to venetoclax based therapy over chemoimmunotherapy.

Asking for predictive markers for every treatment decision may raise the bar too high. Solid data on the prognostic information of genetic markers for different types and lines of treatment should suffice in most settings. Especially, when access to, and tolerability of the proposed therapy are also considered. Mutation testing by NGS, once incorporated into clinical trials, will not only yield rich prognostic information but also provide a more granular resolution of the genetic risk profiles of the enrolled patient population. We are eager to use the tools, we shall learn how to best apply the data they generate to clinical decision making.

\section{Disclosures \\ None}

\section{Funding}

Support received for work outside the submitted work: Adrian Wiestner received research support from Pharmacyclics LLC, an AbbVie company; Acerta Pharma, a member of the Astra-Zeneca group; Merck; Nurix; and Genmab. The author is supported by the intramural research program of NHLBI, NIH.

\section{References}

1. Sutton LA, Ljungstrom V, Enjuanes A, et al. Comparative analysis of targeted next-generation sequencing panels for the detection of gene mutations in chronic lymphocytic leukemia: an ERIC multi-center study. Haematologica. 2021;106(3):662-691.
2.Kipps TJ, Stevenson FK, Wu CJ, et al. Chronic lymphocytic leukaemia. Nat Rev Dis Primers. 2017;3:16096.

3. Burger JA. Treatment of chronic lymphocytic leukemia. N Engl J Med. 2020 0;383(5):460-473

4. Lazarian G, Guieze R, Wu CJ. Clinical Implications of Novel Genomic Discoveries in Chronic Lymphocytic Leukemia. J Clin Oncol. 2017;35(9):984-993

5. Rossi D, Rasi S, Spina V, et al. Integrated mutational and cytogenetic analysis identifies new prognostic subgroups in chronic lymphocytic leukemia. Blood. 2013;121(8):1403-1412.

6. Landau DA, Tausch E, Taylor-Weiner AN, et al. Mutations driving CLL and their evolution in progression and relapse. Nature. 2015;526(7574):525-530.

7. Rossi D, Khiabanian H, Spina V, et al. Clinical impact of small TP53 mutated subclones in chronic lymphocytic leukemia. Blood. 2014;123(14):2139-2147

8. Brieghel C, Kinalis S, Yde CW, et al. Deep targeted sequencing of TP53 in chronic lymphocytic leukemia: clinical impact at diagnosis and at time of treatment. Haematologica. 2019;104(4):789-796.

9. Nadeu F, Clot G, Delgado J, et al. Clinical impact of the subclonal architecture and mutational complexity in chronic lymphocytic leukemia. Leukemia. 2018;32(3):645-653

10. Landau DA, Sun C, Rosebrock D, et al. The evolutionary landscape of chronic lymphocytic leukemia treated with ibrutinib targeted therapy. Nat Commun. 2017;8(1):2185.

11. Ahn IE, Underbayev C, Albitar A, et al. Clonal evolution leading to ibrutinib resistance in chronic lymphocytic leukemia. Blood. 2017;129(11):1469-1479.

12. Blombery P, Anderson MA, Gong JN, et al. Acquisition of the recurrent Gly101Val mutation in BCL2 confers resistance to venetoclax in patients with progressive chronic lymphocytic leukemia. Cancer Discov. 2019;9(3):342-353.

13. Woyach JA, Ruppert AS, Guinn D, et al. BTK(C481S)-mediated resistance to ibrutinib in chronic lymphocytic leukemia. J Clin Oncol. 2017;35(13):1437-1443

14. Ghia P, Stamatopoulos K, Belessi C, et al. ERIC recommendations on IGHV gene mutational status analysis in chronic lymphocytic leukemia. Leukemia. 2007;21(1):1-3.

15. Rawstron AC, Bottcher S, Letestu R, et al. Improving efficiency and sensitivity: European Research Initiative in CLL (ERIC) update on the international harmonised approach for flow cytometric residual disease monitoring in CLL. Leukemia. 2013;27(1):142-149.

16. Rawstron AC, Kreuzer KA, Soosapilla A, et al. Reproducible diagnosis of chronic lymphocytic leukemia by flow cytometry: An European Research Initiative on CLL (ERIC) \& European Society for Clinical Cell Analysis (ESCCA) Harmonisation project. Cytometry B Clin Cytom. 2018;94(1):121-128.

17. Tausch E, Schneider C, Robrecht S, et al. Prognostic and predictive impact of genetic markers in patients with CLL treated with obinutuzumab and venetoclax. Blood. 2020;135(26):2402-2412.

18. Woyach JA, Ruppert AS, Heerema NA, et al. Ibrutinib regimens versus chemoimmunotherapy in older patients with untreated CLL. N Engl J Med. 2018;379(26):2517-2528. 